\title{
Massive Abdominal Aortic Aneurysm Masquerading as Heart Failure
}

\author{
Chase J. Smith ${ }^{\mathrm{a}}$, Wesley M. Jones ${ }^{\mathrm{a}}$, Shishir Mathur ${ }^{\mathrm{b}}$, Sameer Arora ${ }^{\mathrm{a}, \mathrm{c}, \mathrm{d}}$
}

\begin{abstract}
The United States Preventative Task Force recommends screening for all men greater than the age of 65 who have smoked more than 100 cigarettes in their lifetime or have a family history of abdominal aortic aneurysm (AAA). This is largely due to the asymptomatic nature of the presentation of most patients who present with AAA. Symptomatic AAA carries a poor prognosis if not detected in a timely manner. Although abdominal pain remains the most common symptomatic presentation, AAA is notorious to surprise clinicians by its unique masquerading ability. We here present an example of one of these unique presentations. Our case reports a 60 -year-old African American male who presented with $4+$ pitting edema and shortness of breath. After initial clinical assessment, new onset heart failure seemed to be the likely diagnosis. However, the grossly normal nature of echocardiogram forced us to entertain other potential possibilities. A thorough review of the human anatomy and critical multispecialty decision making paved way for intra-abdominal imaging which revealed a massive AAA extending to the common iliac arteries compressing the inferior vena cava. We discuss the existing literature and try to find reasons why diagnosing AAA is still not a perfected art.
\end{abstract}

Keywords: Abdominal aortic aneurysm; Heart failure; Edema; Inferior vena cava

\section{Introduction}

An abdominal aortic aneurysm (AAA) is a dilation of the aorta that develops because of weakening in the vessel wall [1]. The

\footnotetext{
Manuscript accepted for publication July 07, 2016

${ }^{a}$ Campbell University School of Osteopathic Medicine, Lillington, NC, USA bepartment of Cardiology, Cape Fear Valley Medical Center, Fayetteville, NC, USA

'Division of Cardiology, University of North Carolina at Chapel Hill, NC, USA

${ }^{\mathrm{d} C}$ Corresponding Author: Sameer Arora, Division of Cardiology, UNC Chapel Hill School of Medicine, 160 Dental Circle, Chapel Hill, NC 27599-7075, USA.Email: saror@email.unc.edu
}

doi: http://dx.doi.org/10.14740/jmc2582w development of an AAA, classically, begins with an abnormal smooth muscle cell response to inflammatory changes in the weakened vessel [2]. These changes facilitate the development of atherosclerotic plaque, which causes an increased release of proteolytic enzymes such as elastase that further weakens the vessel wall leading to aneurysmal changes [2]. Atherosclerosis, male gender, smoking, increased diastolic pressure, age ( $>$ 60 years) and family history are risk factors for development of AAA [3]. Due to the asymptomatic presentation of most AAAs, the diagnosis is often an incidental finding on imaging [1]. A "symptomatic" AAA usually presents with vague abdominal and/or back pain which is hypothesized to be due to peritoneal inflammation [1]. Due to the lethal nature of the consequences associated with AAA, the awareness of the most unique presentations of this deadly diagnosis is critical to save a valuable human life.

\section{Case Report}

A 60-year-old paraplegic African American male presented with a 4-week history of bilateral lower extremity and scrotal edema. His review of system was positive for shortness of breath accompanied by orthopnea. Past medical and surgical history consisted of a back surgery 30 years ago, which had left him paraplegic with limited motor strength and sensation in his lower extremities. Social history was positive for a 42-pack-year smoking history, but he denied alcohol or illegal drugs. On exam, he was afebrile, tachycardic at 105 beats per minute with a blood pressure 140/88 $\mathrm{mm} \mathrm{Hg}$ and $97 \%$ oxygen saturation on room air. Auscultation of lung revealed bibasilar crackles and cardiac exam showed normal heart sounds with absence of murmurs. Third heart sound was not appreciated. Jugular venous distention was absent. Abdominal exam revealed an obese and protuberant abdomen. No masses were palpable and no bruits were auscultated. Exam revealed 4+ pitting edema extending up to the thighs (Fig. 1). Neurologic exam showed $1 / 5$ power in bilateral lower extremities. Labs showed normal complete blood counts, renal function, and electrolytes. Chest X-ray revealed mildly prominent pulmonary vasculature. Electrocardiogram showed normal sinus rhythm with no abnormalities. Duplex ultrasound of the lower extremity venous system was negative for deep venous thrombosis. On the basis of this presentation, he was diagnosed with 


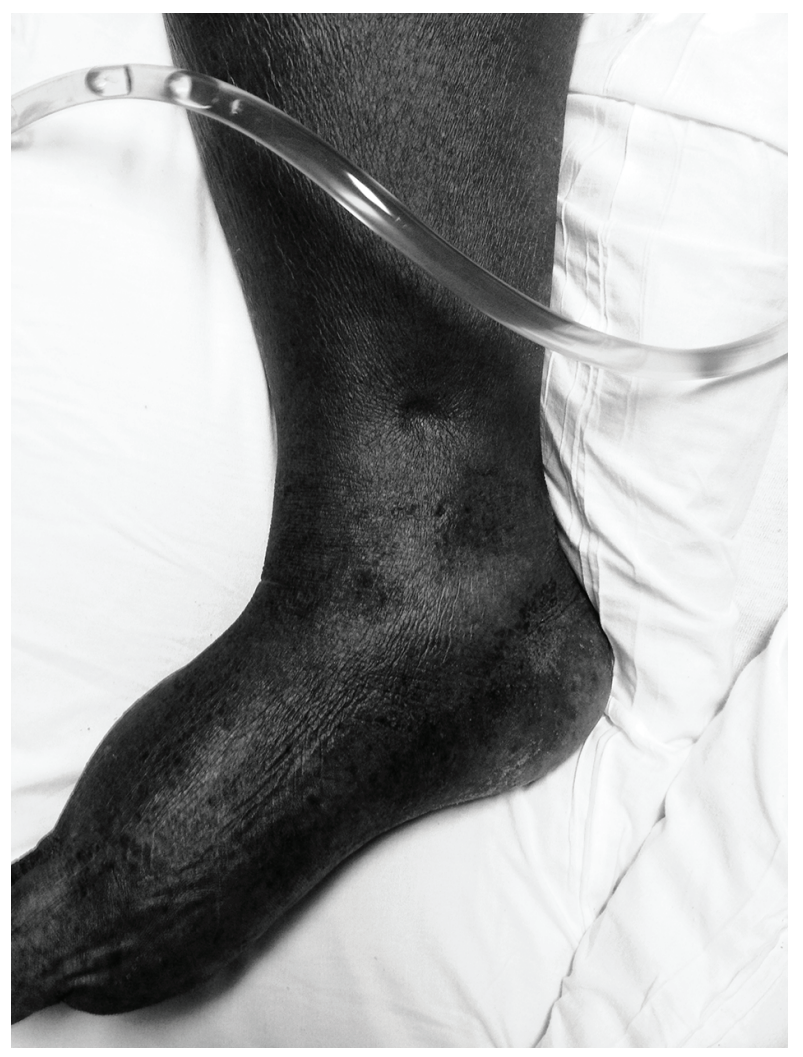

Figure 1. Image showing pitting edema at presentation.

heart failure clinically and an echocardiogram was planned for him the next day. He was started on low dose furosemide for diuresis and low dose lisinopril for afterload reduction.

On day 2, patient underwent an echocardiogram which showed normal left ventricular and right ventricular function with no valvular abnormalities. The findings of the echocardiogram were personally reviewed by the medical team with the cardiologist. After a multi-specialty discussion between the medical and the cardiovascular team, the thought of an intra- abdominal pathology was entertained. Though an abdominal CT scan confirmed the suspicion of an intra-abdominal pathology, the results exceeded expectations. CT showed a large AAA (8.77 cm diameter) (Fig. 2a), extending to involve bilateral common iliac arteries. The right common iliac artery was dilated to $6.16 \mathrm{~cm}$ and the left common iliac artery to $7.27 \mathrm{~cm}$. The inferior vena cava was compressed inferiorly by the iliac aneurysms (Fig. 2b), giving rise to the bilateral lower extremity edema.

The patient underwent an open surgical repair with aortoiliac bypass and tolerated the surgery well. Following the surgical repair, the patient had rapid resolution of lower extremity and scrotal edema. The patient was ultimately discharged to rehabilitation.

\section{Discussion}

Ruptured AAAs are a common and potentially preventable cause of death in the United States. The high mortality stems from the asymptomatic nature of presentation of most AAAs. In 2008, only 5-20\% of surgically corrected AAAs were symptomatic at first presentation [3,4]. Although most cases of AAA are diagnosed as incidental findings, awareness of the atypical ways of presentation is important due to the poor prognosis associated with this diagnosis. A case by Sajjad et al found a large AAA that displaced the inferior vena cava with resulting thromboembolism [5]. Takaseya et al described an aortocaval fistula presenting with unilateral lower extremity swelling [6]. Our patient was stable, in no pain and was not old enough to be screened for AAA. Also, the fact that the initial presentation simulated a classic presentation of new onset heart failure deterred us from the diagnosis at the time of initial presentation.

The US Preventative Task Force recommends screening for all men greater than the age of 65 that have smoked more than 100 cigarettes in their lifetime or have a family history of AAA. The duration of smoking has been shown as an independent risk factor for an increased risk of AAA with a reduc-
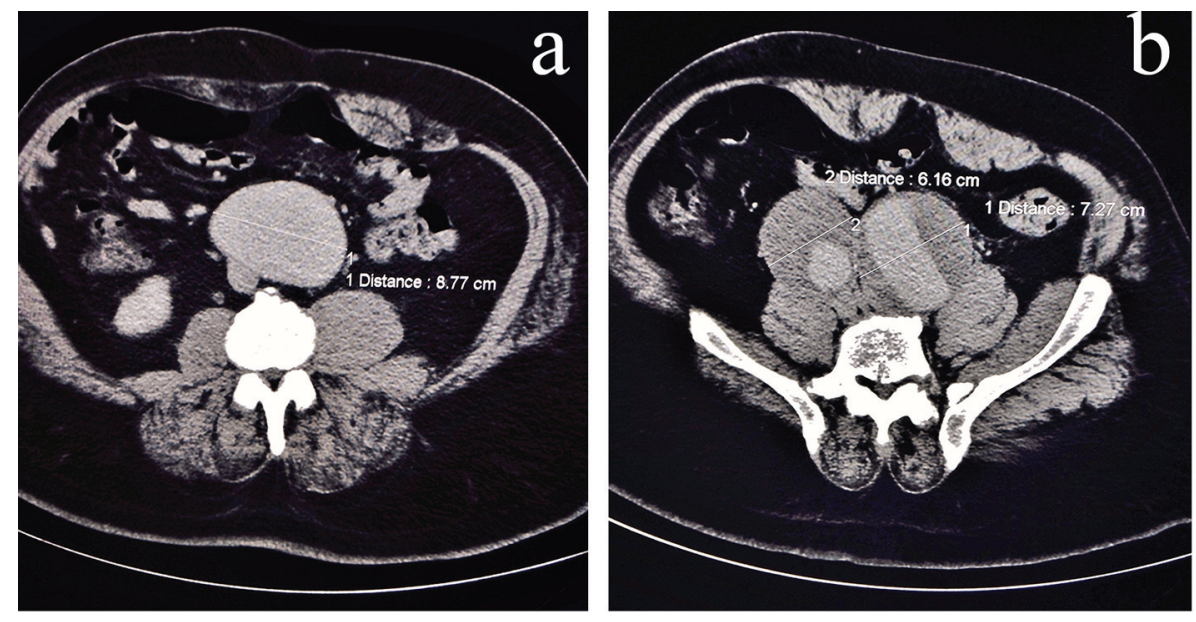

Figure 2. CT abdomen with contrast (axial view). (a) Infrarenal abdominal aortic aneurysm in contact with inferior vena cava (just superior to the nearly complete compression). (b) Common iliac artery aneurysm with venous compression posteriorly. 
tion of risk noted with smoking cessation [7]. Some studies suggest men as young as age 50 would benefit from screening ultrasound for AAA [8]. Recent research suggests that even with a thorough physical exam, the accuracy of abdominal palpation to detect AAA is only $68 \%$ sensitive and $75 \%$ specific. Furthermore, in patients with an abdominal girth greater than $100 \mathrm{~cm}$ (40 inch waistline), this specificity drops to $53 \%$ [9]. Our patient had not been screened for AAA largely due to his age, although he did have multiple risk factors including obesity and an ongoing tobacco use. Our case also brings to question whether there is a space for more autonomy for the physician to screen AAA solely due to the poor prognosis it carries if the diagnosis is not made in a timely manner $[9,10]$.

In conclusion, lower extremity edema developing over a short to intermediate time period with normal cardiac function can be a late presentation of AAA. Diagnosing AAA is challenging and often requires a critical diagnostic decision that can be missed with dire consequences.

\section{Conflicts of Interest}

None.

\section{Funding}

None.

\section{References}

1. Moxon JV, Parr A, Emeto TI, Walker P, Norman PE, Golledge J. Diagnosis and monitoring of abdominal aortic aneurysm: current status and future prospects. Curr Probl Cardiol. 2010;35(10):512-548.
2. Cohen JR, Sarfati I, Danna D, Wise L. Smooth muscle cell elastase, atherosclerosis, and abdominal aortic aneurysms. Ann Surg. 1992;216(3):327-330; discussion 330322.

3. Nevala T, Perala J, Aho P, Matsi P, Ylonen K, Roth WD, Manninen $\mathrm{H}$, et al. Outcome of symptomatic, unruptured abdominal aortic aneurysms after endovascular repair with the Zenith stent-graft system. Scand Cardiovasc J. 2008;42(3):178-181.

4. Sullivan CA, Rohrer MJ, Cutler BS. Clinical management of the symptomatic but unruptured abdominal aortic aneurysm. J Vasc Surg. 1990;11(6):799-803.

5. Sajjad J, Ahmed A, Coveney A, Fulton G. A large unruptured abdominal aortic aneurysm causing pulmonary embolism. J Surg Case Rep. 2015;2015(7).

6. Takaseya T, Hiromatsu S, Akashi H, Okazaki T, Tobinaga $\mathrm{S}$, Aoyagi S. A case of unilateral leg edema due to abdominal aortic aneurysm with aortocaval fistula. Ann Thorac Cardiovasc Surg. 2007;13(2):135-138.

7. Lederle FA, Johnson GR, Wilson SE, Chute EP, Littooy FN, Bandyk D, Krupski WC, et al. Prevalence and associations of abdominal aortic aneurysm detected through screening. Aneurysm Detection and Management (ADAM) Veterans Affairs Cooperative Study Group. Ann Intern Med. 1997;126(6):441-449.

8. Morris GE, Hubbard CS, Quick CR. An abdominal aortic aneurysm screening programme for all males over the age of 50 years. Eur J Vasc Surg. 1994;8(2):156-160.

9. Fink HA, Lederle FA, Roth CS, Bowles CA, Nelson DB, Haas MA. The accuracy of physical examination to detect abdominal aortic aneurysm. Arch Intern Med. 2000;160(6):833-836.

10. Muluk SC, Gertler JP, Brewster DC, Cambria RP, LaMuraglia GM, Moncure AC, Darling RC, et al. Presentation and patterns of aortic aneurysms in young patients. J Vasc Surg. 1994;20(6):880-886; discussion 887-888. 